\title{
El turismo accesible en Chile: articulación y pertinencia de la oferta
}

\author{
Teresa Barrientos Guzmán* \\ Marfilda Sandoval Hormazabal**
}

Recibido 25 de mayo de 2019; aceptado 28 de junio de 2019

\begin{abstract}
There has been an increase in the numbers of disabled persons. The effects of this segment has led to special concern in relation to the tourism sector. The fundamental reason justifying the importance of this niche of the market is its trend towards rapid growth. There is a significant market in our country where access to leisure facilities is required and this, doubtless, should be suitably put together for that end.

Accessible tourism currently constitutes part of the route charted out by public and private organizations. However, and given the characteristics that tourism activity has, it is needed for all the parties involved to participate actively. This makes it possible for tourism in Chile to develop in this country with an inclusive nature and with suitable interventions that belong and should be carried out. So it is of especial relevance for all sectors to encourage accessible tourism, as the first requirement of a disabled person when going out to travel is to be sure that he/she will be able to access physical surroundings adapted to his/her needs.

The bibliography examined and the interviews with users of tourism services have made it possible to obtain information and to make contrasts, providing as a result a first approach towards information that makes clear the progress and the shortfalls or deficiencies that users of inclusive services identify.

Key words: disability, tourism, accessibility, standard, testimony.
\end{abstract}

\section{Resumen}

Existe un aumento de personas con discapacidad, la repercusión de este segmento ha generado una especial preocupación por parte del sector turístico, las razones

* Colegio Especial Jan Van Dijk Corpaliv, correo electrónico: teresa.barrientos@yahoo.es

** Facultad de Administración y Economía, Universidad Tecnológica Metropolitana, correo electrónico: mafi.sandoval@utem.cl 
fundamentales que justifican la importancia de este nicho de mercado es su rápida tendencia al crecimiento. Existe un mercado significativo en Chile que requiere tener accesibilidad a los espacios de ocio, que sin duda deben ser articulados adecuadamente para dicho fin.

El turismo accesible constituye a la fecha, parte de la hoja de ruta de organismos públicos y organizaciones privadas, pero dadas las características que tiene la actividad turística, requiere que participen activamente todos los agentes involucrados, lo cual permita desarrollar el turismo nacional bajo un carácter inclusivo, con la pertinencia adecuada en las intervenciones que se deban realizar, por lo que cobra especial relevancia fomentar el turismo accesible desde todos los sectores, ya que la primera exigencia de una persona con discapacidad al salir de viaje es tener la certeza que podrá acceder a un entorno físico adaptado a sus necesidades.

La bibliografía revisada y las entrevistas a usuarios de servicios turísticos han permitido obtener información y contrastar, entregando como resultado una primera aproximación con información que da cuenta de los avances y carencias que identifican usuarios de servicios turísticos inclusivos.

Palabras claves: discapacidad, turismo, accesibilidad, normativa, testimonio.

\section{Resumo}

Há um aumento de pessoas com deficiência, o impacto deste segmento gerou uma preocupação especial por parte do setor de turismo, as razões fundamentais que justificam a importância deste nicho de mercado, é a sua tendência de crescimento rápido. Há um mercado significativo em nosso país que requer acesso a espaços de lazer, que, sem dúvida, precisam ser articulados adequadamente para esse fim.

O turismo acessível é atualmente parte do roteiro de órgãos públicos e organizações privadas, mas, dadas as características que tem a atividade turística, requer que participem ativamente todos os agentes envolvidos, de maneira a permitir desenvolver o turismo nacional sob uma natureza inclusiva, com a devida pertinência nas intervenções que devem ser realizadas, por isso é particularmente importante promover o turismo acessível em todos os setores, uma vez que o primeiro requisito de uma pessoa com deficiência ao viajar é a certeza de que terá acesso a um ambiente físico adaptado às suas necessidades.

A revisão bibliográfica e as entrevistas aos usuários dos serviços turísticos permitiram obter informações e avalia-las, proporcionando como resultado uma primeira aproximação com informações que mostram os avanços e carências que identificam usuários de serviços inclusivos.

Palavras chave: deficiência, turismo, acessibilidade, normativa, testimunho. 


\section{Introducción}

El turismo se ha convertido en el sector de mayor volumen y más rápido crecimiento del mundo, y sigue creciendo de manera permanente año tras año, debido a tal crecimiento los países están integrando cada vez más al turismo en sus estrategias de crecimiento económicas y desarrollo inclusivo, generando así, mayor empleo y bienestar en las comunidades.

Una infraestructura y equipamiento que no atienda correctamente a las necesidades de las personas con discapacidad y para otros segmentos vulnerables de la ciudadanía, excluye a muchos destinos del mercado. Sin embargo, en muchas ocasiones el entorno no está adecuadamente diseñado para personas con discapacidad, quienes a menudo no pueden disfrutar de la misma manera para viajar que las demás personas.

La Organización Mundial de Turismo (OMT) es consciente de este problema desde que en la Asamblea General de 1991 se aprobaron las primeras recomendaciones dedicadas a la promoción del turismo accesible, revisadas posteriormente en el año 2005.

Es por ello que, en el año 2009, se realizó la Declaración sobre la Facilitación de los Desplazamientos Turísticos, adoptada por la Asamblea General de la OMT, en donde estipulan que el facilitar los viajes turísticos a las personas con discapacidad es un elemento esencial de cualquier política de desarrollo turístico social y ambiental responsable.

Según la Organización Mundial de Salud (OMS, 2011) hay aproximadamente mil millones de personas que tienen alguna discapacidad física, mental o sensorial, a estos datos se suma el envejecimiento de la población, lo cual provoca un aumento en la tasa de discapacidad entre la población con posibilidad de viajar, aumentando así la demanda de entorno, transporte y servicios accesibles, añadiéndole valor al mercado del sector turismo accesible.

En Chile, un $20 \%$ de la población de 18 años o más se encuentra con algún grado de discapacidad, de acuerdo con las cifras entregadas por el Ministerio de Desarrollo Social (2015). Si consideramos que la accesibilidad universal es uno de los elementos clave que deben promoverse para alcanzar la inclusión, se entiende el interés de la Subsecretaría de Turismo de Chile por garantizar el acceso de todas las personas a los atractivos turísticos existentes a lo largo del país. Este tema sin duda se ha transformado en un desafío importante para la administración del Estado. Es por esto que cuando se aborda el desarrollo turístico sustentable en áreas protegidas del Estado, se considera fundamental la variable accesibilidad universal, especialmente al implementar infraestructura pública de soporte para la actividad turística.

Por ello la Corporación Nacional Forestal (CONAF), institución que administra las áreas silvestres protegidas del Estado, lleva a cabo un trabajo de más de dos décadas, enfocado a la implementación de la accesibilidad universal e inclusión social, 
contando a su vez, como soporte, con la Política Institucional que posibilita la continuidad y el progreso de esta importante contribución social, para beneficio de todas las personas que las visitan. A su vez la Corporación lleva a cabo en forma pionera en Chile, la instalación en el quehacer de atención de público en las áreas protegidas, la técnica de guiaje para las terapias de baños de bosque (Shinrin Yoku), medicina forestal tradicional japonesa, que contribuye directamente a mejorar la calidad de vida y salud de las personas, por el contacto pleno con la naturaleza (Lazo, 2019).

Complementariamente y para abordar este tema, la Subsecretaría de Turismo ha trabajado de manera coordinada con la Corporación Nacional Forestal para desarrollar diseños de infraestructura pública con accesibilidad universal, especialmente en senderos, campings, estacionamientos, miradores, baños y áreas de merienda, entre otros.

También se ha dado énfasis en incorporar este tema a nivel público y ciudadano, promoviendo el desarrollo de instancias de discusión a nivel nacional, como lo fue la jornada de capacitación "Diálogos sobre accesibilidad universal en áreas protegidas" (organizada por la Subsecretaría de Turismo y la Corporación Nacional Forestal con el apoyo del Programa de las Naciones Unidas para el Desarrollo, el Servicio Nacional de Turismo y el Servicio Nacional de la Discapacidad, 2016) y el Seminario Internacional sobre Turismo Accesible, realizado el 22 y 23 de septiembre de 2016 (organizado por el Servicio Nacional de Turismo en conjunto con la OMT). Estas instancias buscaron contribuir en la comunicación, sensibilización e incorporación del enfoque de accesibilidad universal y no discriminación, de modo de hacer de Chile un país turístico para todos y todas.

\section{Marco teórico de la discapacidad}

El concepto de discapacidad ha ido cambiando a través del tiempo, ha transitado desde distintos modelos conceptuales, las últimas concepciones estaban basadas en un modelo médico y el modelo social de la discapacidad según plantea la Clasificación Internacional del Funcionamiento y la discapacidad (CIF):

El modelo médico considera la discapacidad como un problema de la persona directamente causado por una enfermedad, trauma o estado de salud (...). Por otra parte, el modelo social de la discapacidad considera la discapacidad como un problema social (...) el manejo del problema requiere la actuación social y es responsabilidad colectiva de la sociedad (Ministerio de Desarrollo Social, Estudio Nacional de la Discapacidad 2015).

Actualmente se ha producido una integración de ambos modelos, llegando al modelo biopsicosocial donde se entiende a la discapacidad como el resultado de las interacciones entre factores que son propios de la persona con discapacidad y factores 
sociales o ambientales. La discapacidad es entonces un concepto multidimensional que abarca la perspectiva biológica, individual y social.

Existen varias formas de agrupar las discapacidades, por ejemplo, de acuerdo con las áreas de funcionamiento afectadas (visión, audición, intelectual, motriz), o de acuerdo al momento en que se produce (genético o adquirido) una de las formas más amplias de hacerlo es como:

- Personas con discapacidad permanente (física, sensorial, intelectual),

- Personas mayores de 60 años sin situación de discapacidad, y

- Personas en situación de discapacidad transitoria, entre los que se incluyen mujeres embarazadas y personas enyesadas, entre otros (ENAT-FUNDACIÓN ONCE, 2015).

El Ministerio de Desarrollo Social, en el Estudio Nacional de la Discapacidad 2015, da cuenta de la metodología para medición de la discapacidad, Figura 1.

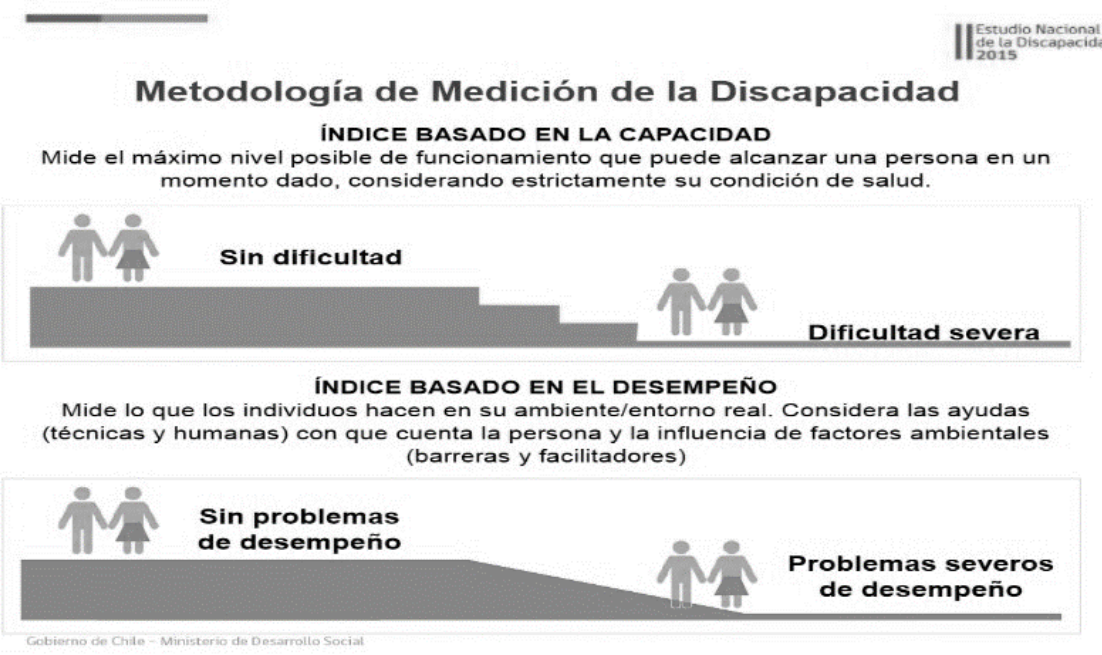

Figura 1. Discapacidad en Chile.

Fuente: Ministerio de Desarrollo Social, Estudio Nacional de la Discapacidad (2015).

Considerando ambas mediciones, es posible estimar que 2836818 personas de 2 y más años $(16.7 \%)$ se encuentra en situación de discapacidad. La Figura 2 ilustra los porcentajes de personas sin discapacidad (PsSD) y personas con discapacidad (PeSD). 


\begin{tabular}{|c|c|}
\hline $\begin{array}{l}\text { Distribución de la Población de NNA, } \\
\text { según situación de discapacidad }\end{array}$ & $\begin{array}{l}\text { Distribución de la Población Adulta, según } \\
\text { situación de discapacidad }\end{array}$ \\
\hline $229904,6 \%$ & $2606904,20 \%$ \\
\hline 3733 281, 94\% & $10421238,80 \%$ \\
\hline PsSD PeSD & —PsSD $\square$ PeSD \\
\hline
\end{tabular}

Figura 2. Comparación de la Prevalencia de discapacidad en Población de Niños, Niñas y Adolescentes (NNA) y Adulta.

Fuente: SENADIS, Estudio Nacional de Discapacidad (2015).

\section{Discapacidad física}

Las personas con discapacidad física son aquellas que presentan una disminución importante en las capacidades de movimiento de una o varias partes del cuerpo en forma permanente o transitoria, también se habla de personas con movilidad reducida (PMR). Es un concepto muy utilizado en accesibilidad, ya que es más amplio que la sola consideración de discapacidad. El grupo de PMR se compone tanto de aquellos que tienen una discapacidad relacionada con la movilidad (paraplejía, tetraplejía, problemas óseos, etc.) como de quienes tienen dificultades por otros motivos (ancianos, embarazadas, personas con niños pequeños, etc.). Al mismo tiempo, no todas las personas con discapacidad presentan movilidad reducida.

\section{Discapacidad intelectual}

La Asociación Americana de discapacidades intelectuales y del desarrollo (AAIDD, antes AAMR) recoge en su $11^{\text {a }}$ edición (2010) la siguiente definición: "La discapacidad intelectual se caracteriza por limitaciones significativas tanto en funcionamiento intelectual, como en conducta adaptativa, tal y como se ha manifestado en habilidades adaptativas, conceptuales y prácticas. Esta discapacidad se origina antes de los 18 años". Este enfoque concibe la discapacidad como el ajuste entre las capacidades de la persona y el contexto en que esta funciona y los apoyos necesarios. 


\section{Discapacidad sensorial}

La discapacidad sensorial según la OMS (2001), “corresponde al tipo de personas que han perdido su capacidad visual o auditiva y quienes presentan problemas al momento de comunicarse o utilizar el lenguaje". La discapacidad visual incluye la baja visión y ceguera, en cambio la discapacidad auditiva se divide en grados de acuerdo con la pérdida auditiva, desde moderada a pérdida total.

\section{Legislación chilena sobre discapacidad}

La discapacidad en Chile se aborda a través de la Ley No 20.422 y trata sobre Igualdad de Oportunidades e Inclusión Social. Esta nueva normativa, tiene como finalidad lograr la plena inclusión social de las personas que presentan discapacidad, asegurando el disfrute de sus derechos, y eliminando cualquier forma de discriminación fundada en ella.

Establece los principios de "vida independiente"; "accesibilidad universal", "diseño universal”; "intersectorialidad"; "participación y diálogo social”, que den cumplimiento a dicha ley.

El artículo $5^{\circ}$ define el concepto de persona con discapacidad, como a "toda aquella que teniendo una o más deficiencias físicas, mentales, sea por causa psíquica o intelectual, o sensoriales, de carácter temporal o permanente, al interactuar con diversas barreras presentes en el entorno, ve impedida o restringida su participación plena y efectiva en la sociedad, en igualdad de condiciones con las demás".

No obstante, es importante estandarizar el lenguaje a usar en este documento, para lo cual se dispondrán las siguientes definiciones en base a la Ley 20.422.

- Ruta accesible: parte de una vereda o de una circulación peatonal, de ancho continuo, apta para cualquier persona, con pavimento estable, sin elementos sueltos, de superficie homogénea, antideslizante en seco y en mojado, libre de obstáculos, gradas o cualquier barrera que dificulte el desplazamiento y percepción de su recorrido.

- Símbolo Internacional de Accesibilidad (SIA): símbolo gráfico conforme a la norma chilena 3180, con silla de ruedas en blanco sobre un fondo azul, Pantone 294C.

- Accesibilidad universal: la condición que deben cumplir los entornos, procesos, bienes, productos y servicios, así como los objetos o instrumentos, herramientas y dispositivos, para ser comprensibles, utilizables y practicables por todas las personas, en condiciones de seguridad y comodidad, de la forma más autónoma y natural posible.

- Diseño universal: la actividad por la que se conciben o proyectan, desde el origen, entornos, procesos, bienes, productos, servicios, objetos, instrumentos, dispositivos o herramientas de forma que puedan ser utilizados por todas las personas o en su mayor extensión posible. 
- Huella podo táctil: recorrido de pavimento con texturas en sobre relieve y contraste cromático respecto del pavimento circundante, destinada a guiar y/o alertar de los cambios de dirección o de nivel en una circulación peatonal.

- Persona con discapacidad: es aquella que, teniendo una o más deficiencias físicas, mentales, sea por causa psíquica o intelectual, o sensoriales, de carácter temporal o permanente, al interactuar con diversas barreras presentes en el entorno, ve impedida o restringida su participación plena y efectiva en la sociedad, en igualdad de condiciones con las demás.

Desde la perspectiva de la Ley 20.423 de Turismo, esta plantea desarrollar y promocionar la actividad turística, por medio de mecanismos destinados a la creación, conservación y aprovechamiento de los recursos y atractivos turísticos nacionales; reconociendo como términos de aplicación el turismo social para referirse a todos los instrumentos y medios a través de los cuales se otorgan facilidades para que las personas, preferentemente de recursos limitados, desarrollen actividades turísticas en condiciones adecuadas de economía, accesibilidad, seguridad y comodidad; y ecoturismo, como la modalidad de turismo ambientalmente responsable, de bajo impacto, que promueve la conservación del medio ambiente y propicia la inclusión activa y socioeconómicamente benéfica de las poblaciones locales.

\section{Avances en accesibilidad universal para el turismo en Chile}

La Accesibilidad Universal según definición que se encuentra en la Ley 20.422, indica que es, "la condición que deben reunir los entornos, procesos, bienes, productos y servicios, así como los objetos e instrumentos, herramientas y dispositivos, para ser comprensibles, utilizables y practicables por todas las personas, en condiciones de seguridad y comodidad, de la forma más autónoma y natural posible" (Ministerio de Vivienda y Urbanismo, Ley 20.422, Decreto $\mathrm{N}^{\circ} 50$ ) Chile ha recogido esta necesidad en diferentes políticas públicas junto a normas relacionadas como la Ley 20.422 sobre la Inclusión Social de las Personas con Discapacidad, la Ley 21.015 sobre Inclusión Laboral, ambas con sus respectivos reglamentos asociados.

En el año 2006, y en el marco del desarrollo de infraestructura accesible, se elabora el Primer Plan de Accesibilidad para las Áreas Silvestres Protegidas del Estado (Boudeguer, 2007). Por otro lado, SERNATUR incorporó en la Estrategia Nacional de Turismo 2012-2020, cinco pilares fundamentales: Promoción, Sustentabilidad, Calidad y Capital Humano, Inteligencia de Mercado, Inversión y Competitividad. En este último se implementó como acción la "Accesibilidad a personas con discapacidad y movilidad reducida" (SERNATUR, 2019), a través del cual se ha estado trabajando en diferentes estrategias asociadas para definir y fortalecer el Turismo Accesible e Inclusivo en Chile, es así, como en el concepto "Turismo 
Accesible" hace referencia a la adecuación de los entornos, productos y servicios turísticos de modo que permitan el acceso, uso y disfrute a todos los usuarios, bajo los principios del Diseño Universal.

Adicional a esto, en el Plan Nacional de Desarrollo Turístico Sustentable, en la Línea de Acción 4, se hace mención del incentivo al turismo interno con enfoque inclusivo.

\section{Avanzando hacia la inclusividad en materia normativa}

El decreto $\mathrm{N}^{\circ} 50$ de marzo de 2016, modifica Decreto $\mathrm{N}^{\circ} 47$ de Vivienda y Urbanismo de 1992, Ordenanza General de Urbanismo y Construcciones en el sentido de actualizar sus normas y disposiciones de la Ley 20.422, sobre igualdad de oportunidades e inclusión social de personas con discapacidad.

En atención a lo anterior, el Servicio Nacional de Turismo (SERNATUR), elabora un documento, con el fin de aclarar la aplicación de la Ordenanza General de Urbanismo y Construcción (OGUC), en materia de accesibilidad y su exigencia para equipamientos del área turística para hoteles, moteles, apart-hoteles, residenciales, pensiones, o similar. En dicho documento se destacan los artículos más relevantes que rigen a las edificaciones relacionadas con el ámbito del turismo. Allí también se recomienda revisar la OGUC, para identificar cómo se clasifica el inmueble, considerando que, a partir de lo anterior, se determinan las exigencias de accesibilidad. Artículo 2.1.25.

El tipo de uso 'Residencial', contempla preferentemente el destino vivienda, e incluye hogares de acogida, así como edificaciones y locales destinados al hospedaje, sea este remunerado o gratuito, siempre que no presten servicios comerciales adjuntos, tales como bares, restaurantes o discotecas, en cuyo caso requerirán que en el lugar donde se emplazan este admitido algún uso comercial...

En consecuencia, si los inmuebles incluyen recintos adicionales como bares, restaurantes etc., pierden la categoría de residencial y se consideran en la categoría comercial (Decreto Supremo N50/2015 Modificaciones OGUC en Materia de Accesibilidad Universal).

\section{El Sistema Nacional de Áreas Silvestres Protegidas del Estado y la Inclusividad.}

La Corporación Nacional Forestal (CONAF), en sus unidades que conforman el Sistema Nacional de Áreas Silvestres Protegidas del Estado (SNASPE), suman 105 unidades a lo largo del país, compuestas por: 41 parques nacionales, 46 reservas nacionales y 18 monumentos naturales. Actualmente 41 de estas áreas protegidas cuentan con servicios accesibles para personas con discapacidad física y sensorial, considerada como un foco de acción institucional respecto a la equidad social y la No 
Discriminación y en el ámbito de la recreación y educación ambiental accesibles en Chile.

La Política de la CONAF es: Las Áreas Silvestres Protegidas del Estado, administradas por la Corporación Nacional Forestal, son para el disfrute, salud, bienestar, conocimiento y valoración de todas las personas sin discriminación por su condición física, mental, sensorial, social, origen étnico, entre otras, en el marco de la inclusión social y la participación ciudadana.

La gran mayoría de estas unidades del SNASPE son visitadas anualmente por millones de personas, por ejemplo, en el año 2017 concurrieron a las áreas: 3019 432 visitantes, de los cuales, 9990 fueron visitantes en situación de discapacidad. Notable incremento (2017) de la magnitud de visitantes de este segmento de la población, al compararlo con el registro ocurrido en el año 2006 (1 330 personas).

Por tales características y demandas del SNASPE, CONAF en forma voluntaria en los inicios de los años 2000 emprendió el desafío de contar con servicios accesibles para todas las personas, fundamentalmente, para el segmento de la población que presenta discapacidad motriz, sensorial y otros, todo ello para el uso y disfrute del patrimonio natural y cultural que contienen las áreas silvestres protegidas.

Hoy en día, 41 de estas áreas protegidas cuentan con elementos de accesibilidad, trabajo que se ha ido concretando progresivamente a contar del año 2002 (Boudeguer, 2007) y por gestión directa de CONAF y con importantes alianzas con el sector público y privado, lo que los ha llevado a ser reconocida como un servicio público con buenas prácticas para el respeto a la biodiversidad y la no discriminación.

Respecto a las acciones de Inclusión Social, el año 2017 la Gerencia de Áreas Silvestres Protegidas en conjunto con los Departamentos regionales de Áreas Silvestres Protegidas, impulsaron y organizaron actividades de visita con inclusión social en al menos una de las áreas protegidas existentes en cada región del país.

En los años 2017 y 2018 han sido beneficiadas aproximadamente 700 personas, principalmente con condiciones de discapacidad (física, sensorial y mental), escuelas con estudiantes en situación de pobreza, adultos mayores y otros. Este tipo de servicio se replicará cada año en las áreas silvestres protegidas (ASP), beneficiando al menos 300 a 400 personas por año y procedentes de segmentos vulnerables de la población chilena, como personas con discapacidad, movilidad reducida, en situación de pobreza, riesgo social, enfermos terminales, y otros.

Con esta realidad y sabiendo que entre los objetivos que han motivado la creación y manejo de las áreas silvestres protegidas del Estado, se contempla los propósitos de: promover y facilitar las oportunidades de esparcimiento en contacto con la naturaleza y cultura que ellas contienen y - que se suma a la creciente demanda por uso de estos espacios naturales protegidos de la Nación- se comienza con la Coconstrucción de la Estrategia Nacional de Accesibilidad Universal a la Naturaleza a través del SNASPE y su entorno (ENAUN). 
Esta corresponde a una gestión que CONAF decidió a contar del año 2017 con la finalidad de dar un mayor impulso al trabajo de accesibilidad e inclusión social, pero con la necesaria e importante participación del sector público, privado y los propios beneficiaros.

Los aspectos esenciales que se pueden rescatar de dicha iniciativa es la coconstrucción con personas con discapacidad y de otros segmentos de la población chilena, donde además participan en forma protagónica servicios públicos y privados como por ejemplo: CONAF, MINAGRI, SENADIS, MINSAL, SERNATUR, Subsecretaría de Turismo, INJUV, TELETÓN, Pontificia Universidad Católica de Chile (Escuela de Diseño), Universidad Tecnológica Metropolitana (programa CECAT), Fundación ERES, Fundación Sendero Chile, consultoras como Justo\&Necesario, consultora ASIDES Ltda, DOI-ITAP del Ministerio del Interior de Estados Unidos, y otras organizaciones que se han ido incorporando a este trabajo de servicio social y contribución al bienestar humano asociado a naturaleza.

Con la Estrategia Nacional de Accesibilidad Universal a la Naturaleza a través del SNASPE y su entorno (ENAUN), se espera contribuir al fortalecimiento de políticas públicas en salud y del bienestar humano integral para todos (a) los habitantes del territorio nacional.

Por último, la CONAF, ha desarrollado durante el 2017, la Estrategia Nacional de Accesibilidad Universal para las Áreas Silvestres Protegidas, con el objetivo de entregar "Naturaleza para todas las personas" (Lazo, 2018).

Es importante, reiterar los casi tres millones de personas con situación de discapacidad, (SENADIS, 2015). Esto significa que un $16.7 \%$ de la población nacional mayor de dos años tenía algún impedimento de acceso universal a cualquier actividad en el sector turístico. Es por esto que cuando se aborda el desarrollo turístico sustentable en áreas protegidas del Estado, se considera fundamental la variable accesibilidad universal, especialmente al implementar infraestructura pública de soporte para la actividad turística.

En SERnATUR, en el marco del Programa de Conciencia Turística que aborda temas de ética y responsabilidad social y recogiendo lo estipulado en el Código de Ética Mundial para el Turismo, existe un área de Turismo Accesible, la cual tiene como función implementar acciones para que los turistas con discapacidad y sus acompañantes puedan acceder y disfrutar de la oferta turística nacional de forma autónoma, cómoda y segura.

Además, se preocupa que la accesibilidad esté presente, de manera trasversal, en todas las áreas del turismo nacional para permitir el acceso a todas las personas por igual. Así mismo, estimula la generación de productos turísticos diseñados bajo el concepto de accesibilidad universal y del Código de Ética Mundial para el Turismo. Entre los prestadores turísticos, se encuentran iniciativas dedicadas al turismo enológico y cultural; turismo aventura, con trekking, cabalgatas y expediciones; 
turismo geográfico e histórico con senderismo inclusivo; hotelería y cabañas con adecuaciones accesibles, restaurantes, entre otros.

Iniciativas destacadas durante el año 2018 en Turismo accesible, se encuentran el "Piloto de accesibilidad para productos y atractivos turísticos", en el cual SERNATUR, consciente de la importancia que ha adquirido la accesibilidad en el desarrollo del turismo y desde su compromiso por la inclusión de las personas con discapacidad, toma la iniciativa de poner en marcha un proyecto para la ciudad de Santiago, un piloto de accesibilidad para servicios y atractivos turísticos como herramienta para impulsar la accesibilidad en el turismo en el resto de los destinos que el país dispone. El objetivo principal de este proyecto fue definir un "modelo de creación de destino turístico para todos" dentro de Chile y que fuese un referente de inclusión en Latinoamérica (SERNATUR, 2019) ${ }^{1}$.

Se trabaja una metodología donde se logre establecer, el nivel de accesibilidad idóneo que debe cumplir un atractivo y los servicios turístico, para ser considerados accesibles, para lo cual es fundamental conocer la situación real de los atractivos y servicios turísticos de Santiago en materia de accesibilidad, que permita detectar aquellos atractivos y servicios turísticos que pueden ser considerados como accesibles y potencialmente accesibles, con ello mejorar la información en materia de accesibilidad del destino turístico Santiago.

El proyecto contempló dos etapas, en la primera se creó un modelo de evaluación de la accesibilidad en base a un catálogo de criterios que sirvieron como referente metodológico para el diagnóstico del grado de accesibilidad de los atractivos y servicios turísticos. Esta etapa del proyecto se realizó en base a una metodología desarrollada "exprofeso" para el mismo y que incluyó varias referencias legislativas y normativas de aplicación en el campo de la Accesibilidad Universal, OGUC de Chile, el estándar internacional de accesibilidad en edificios ISO 21542:2011, además de otra legislación relacionada y la experiencia y conocimiento de los expertos en accesibilidad de ILUNION Tecnología y Accesibilidad.

En tanto para la segunda etapa, se aplicaron los instrumentos de evaluación definidos en diferentes servicios y atractivos turísticos de Santiago. El objetivo de esta etapa fue validar el modelo de evaluación de la accesibilidad propuesta, analizar las brechas para cada ficha aplicada y realizar una propuesta de rutas turísticas en base a los instrumentos analizados con un plan de trabajo para su puesta en valor.

Otra de las iniciativas que se han desarrollado se relaciona con la identificación de los "Lineamientos Nacionales Turismo Accesible", con el objetivo de generar una base estratégica nacional público privada, con miras al desarrollo de este sector.

La metodología para la generación de los lineamientos se basó en tres ejes principales por los que SERNATUR ha estado trabajando, y tienen relación con el 
Capital Humano, Infraestructura y Entornos Habilitantes Accesibles como base para el desarrollo de un destino accesible e inclusivo. Inversión en infraestructura, información y capacitación y la asociatividad, que permita identificar y articular actores relevantes y fortalecer la interacción entre pares, con el fin de otorgar mejoras a un amplio segmento de la población y sumar redes de colaboración.

Las jornadas de trabajo fueron participativas con actores del ámbito público y privado, afines a la accesibilidad. Esta metodología de trabajo será utilizada como un referente en las regiones, también sirvió de insumo base para elaborar la Estrategia Nacional de Turismo Accesible, la cual será la carta de navegación en materia de Turismo Accesible para Chile, considerado como la base y guía para las acciones que se deberán ejecutar a nivel nacional ya que fue validada ante actores claves públicos y privados, en diciembre de 2018.

\section{Los desafios para desarrollar el turismo con Accesibilidad Universal en Chile}

El trabajo realizado para potenciar la industria del turismo nacional ha recibido diferentes reconocimientos como, por ejemplo, el mejor destino de turismo aventura en cuatro oportunidades por World Travel Awards. Chile acaba de ser catalogado por Lonely Planet, una de las mayores guías de turismo mundial, como "Destino Imperdible 2018”, superando a otros nueve países como Sudáfrica, China y Malta, tal como recoge su última publicación Best in Travel, 2018 (SERNATUR, 2019).

Lo anterior, obliga a seguir avanzando en el desarrollo de un turismo que sea más inclusivo, que permita generar nuevas y mejores experiencias en esta actividad, incrementar los destinos con un sello territorial y, a la vez, mejorar la calidad de los servicios, asociadas a equipamiento, infraestructura y capital humano.

Se deben diseñar ciudades pensando en abordar el desafío de la diversidad humana, no solo las necesidades de las personas con discapacidad, también de los niños menores de 8 años y las personas mayores de 80. Ciudades 8-80: El objetivo de esta medida es avanzar en un plan de accesibilidad universal para que en un plazo de 10 años los espacios públicos (incluidos, semáforos, aceras etc.), áreas verdes, edificios y transporte público y privados de nuestras ciudades sean $100 \%$ accesibles a personas con discapacidad, niños y adultos mayores. Para ello, entre otras cosas, se ha trabajado para disponer de un Catastro Nacional de Accesibilidad, desarrollando un sistema de diagnóstico para edificios de uso público que permita dar cuenta del cumplimiento de estándares de accesibilidad, partiendo por instituciones públicas de la Administración del Estado y municipios en todas las comunas y promover las adecuaciones que se deben realizar. Lo anterior permitirá avanzar hacia un Chile Destino Inclusivo, donde se consideren entornos naturales y urbanos, servicios, infraestructura y transporte accesible en todas las regiones (SERNATUR, 2018).

Los entornos habilitantes se consideran como la base concreta para sustentar el turismo accesible. Sin accesibilidad, no hay inclusión. Por lo tanto, uno de los 
esfuerzos que debe prevalecer desde SERNATUR es el catastro de actividades y promover su locación, entendiendo que SERNATUR no es el responsable de la construcción y fiscalización de este tema (SERNATUR, 2019). También el capital humano se requiere en el corto plazo avances significativos en temas de inclusión y accesibilidad en el turismo, además la asociatividad permitirá articular adecuadamente la oferta accesible de un destino.

Por último, y no menos importante es la incorporación de tecnologías y la innovación son temas que replican la necesidad de personas con discapacidad de tener acceso a la información de manera oportuna y en formatos accesibles.

\section{El desafio de crear espacios accesibles para personas ciegas y de baja visión}

En el año 2014 la Universidad Tecnológica Metropolitana (Universidad Pública del Estado de Chile) a través del Departamento de Cartografía y el Programa Centro de Cartografía Táctil (CECAT), junto al Instituto Panamericano de Geografía e Historia (IPGH-OEA), desarrollaron el proyecto de investigación "Propuesta de estandarización de simbología táctil para Latinoamérica: aplicación en cartografía turística", en el cual participaron investigadores de Chile, Brasil y Perú, y que han estado trabajando en conjunto desde 1994, y han conformado un equipo de profesionales interdisciplinario que incluye las áreas de cartografía, geografía, diseño, turismo y educación diferencial, los cuales evaluaron el "Manual para la construcción de cartografía y uso de símbolos táctiles", generando sugerencias del proceso de normalización y estandarización para la simbología táctil en turismo.

Esta iniciativa contribuyó en nuestro país a operacionalizar acciones que contemplan instrumentos de planificación asociados al Plan Estratégico de Accesibilidad Turístico de Chile (SERNATUR), así como también con la Estrategia Nacional de Accesibilidad Universal a la Naturaleza a través del SNASPE y su entorno (ENAUN), liderado por la CONAF, y el aporte entregado por este proyecto, sin duda fue muy importante y sentará un precedente para otros países, al no existir hasta el momento este tipo de mapas estandarizados para turismo.

El mapa táctil es una herramienta que ayuda a lograr una mejor representación mental del espacio, tanto para la persona ciega como para la persona vidente. Complementa los conocimientos memorísticos obtenidos al mostrar el mapa un área o un espacio tridimensional y las posiciones de los elementos que lo integran.

Permite una educación en igualdad de condiciones con un máximo desarrollo de las capacidades y destrezas direccionadas a lograr un máximo desarrollo de la sociabilidad. A través de su utilización se accede a la realización de procesos mentales importantes como reconocer, identificar, comparar, analizar, transferir y complementar la información. Además, fortalece la adquisición en el conocimiento de orientación (puntos cardinales), lateralidad (adelante, atrás, al lado, al frente), y ayuda a discriminar formas y tamaño (Coll, Barrientos, 2017). 
Lo anterior permite entregar un aporte y avanzar hacia la inclusividad, dando la posibilidad a un segmento que muchas veces está totalmente al margen de lo que es la actividad turística. Esta iniciativa les permite a las personas que puedan hacer sus mapas mentales, cuando se hace referencia a un lugar, lo imaginen, pero las personas ciegas mientras no tocan, no tienen esa concepción del espacio.

\section{Resultados de entrevistas realizadas a usuarios con discapacidad visual de servicios turísticos en Chile}

Con el afán de poder identificar la pertinencia que tienen las iniciativas asociadas al equipamiento, infraestructura y recurso humano que se encuentran prestando servicios al segmento de discapacitados en Chile, es importante puntualizar en aquellos temas que resultan relevantes a considerar, para lo cual se aplicaron entrevistas a usuarios discapacitados visualmente que han hecho uso de servicios turísticos a nivel nacional en Chile, que nos permitieran además validar la pertinencia del trabajo desarrollado desde el Centro de Cartografía Táctil, con la "Propuesta de estandarización de simbología táctil para Latinoamérica: Aplicación en cartografía turística".

Tal actividad se realizó en un formato de entrevista, cuya estructura se presenta a continuación:

- Formato de entrevista a usuarios discapacitados

- Pauta de entrevista: es una conversación de carácter abierto y, además de las preguntas que se van haciendo, se pueden ir contando cosas que se consideran importantes para esta investigación.

- Objetivo general:

- Identificar Brechas (aciertos y necesidades), asociadas a personas con discapacidad que realizan turismo y que en la actualidad no les permite desarrollar adecuadamente la actividad turística.

- Objetivos específicos:

- Identificar las dificultades y las satisfacciones de personas con discapacidad que son usuarios de los servicios asociados a la actividad turística en Chile.

- Priorizar necesidades que les permita desarrollar de mejor forma la actividad turística

- Información solicitada

- Datos biográficos

- Métodos de información y de reserva

- Medios de transporte utilizados

- Frecuencia de viajes realizados

- Tipos de turismo que prefiere

- Tipo de alojamientos utilizados

- Dificultades encontradas

- Grado de satisfacción con las propias experiencias como turista. 


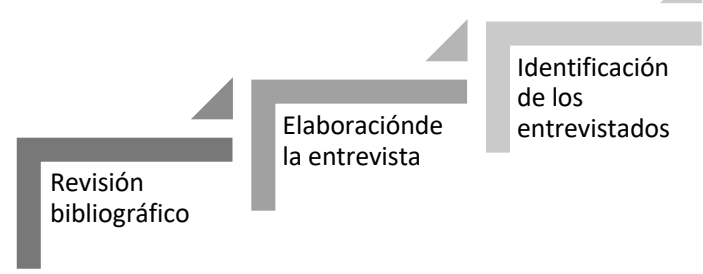

Aplicación de

las entrevistas

Análisis de la

recopilada

Figura 3. Pasos metodológicos.

\section{Aplicación de la entrevista}

En el marco de esta investigación se pudo entrevistar a trece usuarios de servicios turísticos y para lo cual se caracteriza dicho perfil como hombres y mujeres de entre 20 y 40 años, profesionales y deportistas, que han viajado dentro y fuera del país, quienes han indicado que sus experiencias asociadas a la oferta de servicios turísticos en nuestro país, les es poco confiable, por la incertidumbre que les significa llegar a espacios en los cuales están ofertados para personas con discapacidad, pero que finalmente no cumplen su función, o solo existe en número muy reducido, que están solo en lugares puntuales, pero para desplazarse y llegar no están las condiciones adecuadas.

\section{Análisis de la información de la entrevista}

- Transporte: Por ejemplo, uno de los entrevistados indicó que, al viajar en el metro, no siempre anuncian las estaciones, la calidad y el tipo de información que entregan, por lo que indican que se requiere estandarizar información. Otra entrevistada manifestó que en el Metro su perro guía ha sido agredido por pasajeros que lo patean mientras el tren está en movimiento o que hay personas que "llaman al perro" desde el andén del frente poniendo en riesgo a la persona usuaria de perro guía. Estas situaciones descritas ponen de manifiesto una falta de cultura en la población en relación con la importancia de respetar a las personas con discapacidad y reconocer en el perro guía los ojos del usuario para desplazarse con seguridad. También se indicó que en el pasamanos a veces se encuentra información en Braille que indica cantidad de escalones, pero sería de mayor utilidad si indicara el sector de salida (Bike, 2016). En los paraderos de micro sería de utilidad códigos QR, en un lugar estándar, que informen las micros que pasan por ese paradero los cuales pueden ser leídos por los celulares. Con respecto al transporte aéreo se reporta que las líneas aéreas en general carecen de instructivos en Braille, solo American Airline y LATAM disponen de dicho instructivo de seguridad. 
- Tipo de turismo: En general sus viajes son realizados en compañía de familiares, en otros casos como parte de un equipo deportivo. Esto también repercute en que información y reserva sean tareas encargados a otras personas.

- Sitios de interés: museos, parques, restoranes, conciertos. Se destaca que en el Museo de Artes Visuales (MAVI), existe audio descripción para los cuadros y esculturas, en otros lugares de la ciudad esto puede ser imitado o en su defecto poner placas en Braille con la denominación de las esculturas por ejemplo del Parque de las Esculturas en Providencia. Se requiere de mayor publicidad en las redes sociales de los lugares que tienen accesibilidad como el Parque Inés de Suarez de Providencia que cuenta con imágenes táctiles con información de los árboles que allí existen.

- Servicios: cuando ha habido la oportunidad de tener acceso a guía turístico, es difícil para ellos preocuparse de la persona con discapacidad en especial, depende mucho de su sensibilidad y conocimientos para describir lo que se está viendo o lo que las personas están haciendo. Las personas entrevistadas han coincidido en expresar que los guías turísticos debieran disponer de elementos que contribuyan a una contextualización espacial, tener alguna formación que les facilite por ejemplo describirles situaciones geográficas interesantes como los geiseres del norte, edificios u obras de arte.

Una de las entrevistadas que ha viajado a estudiar a España, indica que la Organización Nacional de Ciegos Españoles (ONCE), entrega una tarjeta a sus asociados, con la cual obtiene rebajas en los establecimientos vinculados a cadenas de restaurantes, hoteles, empresas de transporte, lo cual constituye una motivación para que ellos viajen, pero que además ellos no van solos por lo que finalmente termina siendo una forma de promover ciertas lugares o destinos turísticos. Reafirma lo planteado por otro entrevistado de la falta de establecimientos que dispongan de un servicio que respondan a las necesidades de los usuarios, destaca que la huella podo táctil de la línea 4 del Metro de Santiago, Campus San Joaquín está bien elaborada y cumple muy bien su función, no así otras que en vez de ayudar, terminan confundiendo a sus usuarios.

También indicaron que son muy pocos establecimientos que disponen de carta en braille y que su personal se comunique con lenguaje de señas o que sepan tratar a una persona ciega. Se destacó que al menos tres restaurantes visitados cuentan con carta en Braille: Club de Jazz Santiago (Ñuñoa), la Picola Italia y la Sanguchería de Ñuñoa. Por otra parte, indican que los establecimientos no disponen de su carta en tamaños de letras que las personas de baja visión pueden leer.

En Santiago y en Concepción se reconocen avances como semáforos con sonido que apoyan el cruce de calles o la huella podo táctil o texturas para guiar sus recorridos, pero se producen dificultades con los vendedores ambulantes que ocupan espacios sobre las guías, así como también la música con parlantes que confunde el sonido de los semáforos. 
Ninguno de los entrevistados reporta haber recibido algún mapa con información del lugar visitado, lo cual consideran fundamental el disponer de una representación mental del espacio que visitan, ya que les permite la realización de procesos mentales importantes como reconocer, identificar, comparar, analizar, transferir y complementar la información. Además, fortalece la adquisición en el conocimiento de orientación (norte, sur, este y oeste), lateralidad (adelante, atrás, al lado, al frente), y ayuda a discriminar formas y tamaño.

Destacan las iniciativas que se desarrollan al interior de espacios protegidos, donde se están abriendo nuevas y significativas experiencias en un esfuerzo entre diferentes entidades públicas como privadas, destacan la labor realizada por la CONAF como articulador de iniciativas que han sumado organismos como el Instituto Nacional de la Juventud (INJUV), SENADIS, Fundación Sendero de Chile, Fundación ERES, entre otras, que les ha permito construir senderos inclusivos y organizar actividades de voluntariado, las cuales se requieren sumar recursos humanos y económicos, que se sostengan en el tiempo y que se institucionalicen.

Sugerencias realizadas por los entrevistados:

- Solicitan que consideren desarrollar modelos a escala de los lugares patrimoniales o históricos para conocerlos.

- Hay espacios abiertos que les gustaría visitar, pero los evitan, por encontrarse sucios con basura y excremento de animales y que terminan con un equipamiento que no puede ser utilizado, por ejemplo, el Parque Inés de Suarez en Providencia, que resulta ser una interesante apuesta, pero difícil de poder ser utilizada por los usuarios para quienes fue construida.

- Con respecto al equipamiento e infraestructura, recurso humano que tienen los establecimientos asociados a la planta turística, requieren mejorar, dado que hay establecimientos que indican ser accesible, pero finalmente no reúnen las condiciones. El equipamiento básico también está con muchas carencias que aún no se han subsanado, por ejemplo, los cajeros automáticos, no han sido adaptados para el uso de una persona con discapacidad visual.

- Consideran que se hace necesario difundir más aquellos lugares que ya cuentan con accesibilidad a través de las redes sociales y medios de comunicación, así podrían ser incorporados en la APP Lazarillo que está siendo muy utilizada para los desplazamientos.

\section{Conclusiones}

En lo que respecta el turismo accesible, en la actualidad Chile se enfrenta a un gran desafío, que corresponde a la coordinación y suma de los agentes llamados a ser parte desde todos los ámbitos: público, privado, sociedad civil, academia, entre otros, que permita avanzar como país, tanto en infraestructura, equipamiento, empleabilidad de personas en situación de discapacidad, como en empatía, trato digno e igualdad de oportunidades. En la Agenda 2030 para el Desarrollo Sostenible, se establece que la 
discapacidad no puede ser un motivo o criterio para privar a las personas de acceso a programas de desarrollo y el ejercicio de los derechos humanos.

El Turismo Accesible e Inclusivo no se limita a la eliminación de barreras físicas, sensoriales o de la comunicación, sino que tiene por finalidad lograr que los entornos, atractivos y servicios turísticos puedan ser disfrutados en igualdad de condiciones por cualquier persona con o sin discapacidad.

Se requiere socializar a la industria turística con iniciativas asociadas a este nicho. El Sello Inclusivo entregado por SENADIS, a pesar de ser una gran iniciativa, en el sector turístico no refleja cambios debido a la falta de conocimiento de esta información, lo cual indica que debe tener mayor difusión y socialización con los oferentes de los servicios.

En base a las entrevistas desarrolladas, se reconocen los avances que han propiciado desde los organismos públicos y que los privados han tenido que responder, pero cualquier adaptación de equipamiento, infraestructura y capacitación del recurso humano, sugieren los entrevistados que se haga con personas que finalmente serán los usuarios para que realmente cumpla con las reales necesidades que ellos tienen.

La atención hacia el segmento del turismo accesible representa un desafío para el sector turístico en Chile, en términos de mejorar los entornos y movilizar la inversión, que permitan articular la oferta adecuada para este segmento.

En ese ámbito, se reconoce la importancia que tiene el disponer de un mapa táctil que ayude a lograr una mejor representación mental del espacio visitado o que se desea visitar, en el entendido que ello también permitirá programarse adecuadamente tanto para la persona ciega como para la persona vidente. Complementa los conocimientos memorísticos obtenidos al mostrar el mapa un área o un espacio tridimensional y las posiciones de los elementos que lo integran.

Finalmente, se reconoce que el avance logrado en Chile en esta materia, comenzó por personas que han tenido la fuerza y convicción que los ha llevado a sumar esfuerzos tanto humanos como económicos, a la fecha, Chile tiene trazada sus políticas desde el ámbito Público para desarrollar el turismo inclusivo, existen empresas privadas y fundaciones que se han sumado, existe un potencial turístico y una población con discapacidad, ansiosa de disfrutar del turismo, las condiciones están dadas para seguir avanzando, pero se requiere articular desde los diferentes ámbitos los componentes de un producto turístico, que finalmente satisfaga las necesidades de las personas con discapacidad, se requiere planificar para personas discapacitadas y con personas discapacitadas, como protagonistas de sus necesidades.

\section{Bibliografía}

Boudeguer, A. (2007). Manual de Diseño Accesible en áreas silvestres protegidas del Estado, Corporación Forestal Nacional, Chile. Recuperado de 
$<$ http://www.subturismo.gob.cl/wp-content/uploads/2016/09/ManualAccesibilidad-SNASPE.pdf $>$.

Cámara Interamericana de Transporte (2015). "Entorno turístico". Recuperado de $<$ http://www.citamericas.org/arquivos/8eb24c8f67879c1b5b9bfdef68ba48f2.pdf $>$.

Chile. Consejo de Transparencia (2011). Instructivo General $N^{\circ} 10$ del Consejo para la Transparencia sobre el Procedimiento Administrativo de Acceso a la Información. Recuperado de <http://archives.cplt.cl/artic/20121223/asocfile/201212 23235850/instruccion_general_10_dai.pdf $>$.

Chile. Ministerio de Desarrollo Social (2015). Encuesta del Segundo Estudio Nacional de la Discapacidad. Recuperado de <https://www.senadis.gob.cl/pag/137/1398/introduccion>.

Chile. Ministerio de Vivienda y Urbanismo, Ley 20.422, Decreto $N^{\circ} 50$.

Chile. Municipio de Santiago (s.f.). "Entorno turístico". Recuperado de $<$ http://www.santiagocapital.cl/categorias/vermapa/>.

(2016). "Entorno turístico". Recuperado de <http://www.santiagocapital.cl/ fichas/home/plaza-de-armas/parques-y-plazas/>.

Coll, A.; Barrientos, T. y Huentelemu, V. (2017). Enseñando y aprendiendo el espacio geográfico por medio del tacto: orientaciones metodológicas, Ediciones Universidad Tecnológica Metropolitana, Santiago de Chile.

Comuna de Santiago (2016). Estacionamiento reservados para personas con discapacidad. Recuperado de <www.santiagocapital.cl $>$.

Federación Provincial de Asociaciones de Personas con Discapacidad Física y Orgánica de Jaen (FEJIDIF) (s.f.). Trato adecuado a las personas con discapacidad 2010. Recuperado de <www.fejidif.org/>.

Huesca González, A., Ortega Alonso, E. (2004). Hábitos y Actitudes hacia el turismo de las personas con discapacidad física. Informe de resultados 2004. Editor: Plataforma Representativa Estatal de Discapacitados Físicos-Predif. Recuperado de $<$ http://riberdis.cedd.net/handle/11181/4158>.

Lazo, A. (2019). SHINRIN YOKU: Medicina a través de Baños de Bosque para el bienestar humano y beneficio para la salud de todas las personas.

- (2018). Minuta sobre gestión de accesibilidad para las Personas con discapacidad, adultos mayores y otros segmentos vulnerables de la población en las Áreas Silvestres Protegidas del Estado en Chile.

Ley No 20.422 y Trata sobre Igualdad de Oportunidades e Inclusión Social.

ONCE y SFSM (s.f.). Guía de accesibilidad a los medios de comunicación de personas con discapacidad sensorial. Recuperado de <http:/guiacomunicacion. lgperea.com/>.

Organización Mundial de la Salud (2011). "Discapacidad mundial”. Recuperaado de $<$ http://www.who.int/disabilities/world_report/2011/summary_es.pdf >. 
Organización Mundial de Turismo (2011). La OMT y sus asociados promoverán el turismo accesible para todos. Recuperado en $<$ http://www2.unwto.org/es/pressrelease/2011-09-28/la-omt-y-sus-asociados-promoveran-el-turismo-accesiblepara-todos $>$.

—, (s.f.). "Turismo accesible". Recuperado de $<$ http://ethics.unwto.org/es/ content/turismo-accesible>.

SENADIS (2014). Contenidos accesibles para personas con discapacidad. Recuperado de $<$ www.senadis.cl $>$.

SENATUR. Subdirección de Desarrollo (2018). Informe de gestión año 2018. Conciencia Turística-Turismo Accesible. Recuperado de <https://biblioteca.sernatur.cl/ documentos/153.796.18\%20S491i.2019.pdf>.

(2019). Estrategia de Turismo Accesible.

Universidad de Salamanca-Instituto Universitario de Integración en la Comunidad (2016). Servicio de información sobre la discapacidad. Recuperado de $<$ https://sid.usal.es/56036/1-1>.

- (s.f.). Servicio de información sobre la discapacidad. Recuperado de $<$ http://sid.usal.es/2795/9-10>.

(2015). Servicio de información sobre la discapacidad. Recuperado de $<$ http://sid.usal.es/2796/9-10>.

(2016). Servicio de información sobre la discapacidad. Recuperado de $<$ http://sid.usal.es/54807/1-1>. 